\title{
Use of Circulating and Cellular miRNAs Expression in Forensic Sciences
}

\author{
Raluca Dumache1, Alexandru Florin Rogobete2,3, Dorel Sandesc ${ }^{2,3}$, Ovidiu Horea Bedreag 2,3 , \\ Veronica Ciocan1, Camelia Muresan, Adrian Tudor Stan¹, Mihai Sandesc', Anca Dinu', Sonia Elena \\ Popovici', Alexandra Enache ${ }^{1}$ \\ 1 Department of Forensic Medicine, "Victor Babeș” University of Medicine and Pharmacy, Timișoara, Romania \\ 2 Faculty of Medicine, "Victor Babeș" University of Medicine and Pharmacy, Timișoara, Romania \\ ${ }^{3}$ Clinic of Anesthesia and Intensive Care, "Pius Brinzeu" Emergency County Hospital, Timișoara, Romania
}

\section{CORRESPONDENCE}

Alexandru Florin Rogobete

Bd. Iosif Bulbuca nr. 10

300736 Timișoara, Romania

Tel: +40356433111

E-mail: alexandru.rogobete@umft.ro

\section{ARTICLE HISTORY}

Received: July 27, 2017

Accepted: August 17, 2017
Raluca Dumache • Piața Eftimie Murgu nr. 2, 300041 Timișoara, Romania. Tel: +40 256204400 Dorel Sandesc • Bd. Iosif Bulbuca nr. 10, 300736 Timișoara, Romania. Tel: +40 356433111 Ovidiu Horea Bedreag • Bd. losif Bulbuca nr. 10 300736 Timișoara, Romania. Tel: +40 356433111 Veronica Ciocan • Piața Eftimie Murgu nr. 2, 300041 Timișoara, Romania. Tel: +40 256204400

Camelia Mureșan • Piața Eftimie Murgu nr. 2, 30004 Timișoara, Romania. Tel: +40 256204400

Adrian Tudor Stan • Piața Eftimie Murgu nr. 2, 30004 Timișoara, Romania. Tel: +40 256204400

Mihai Sandesc • Piața Eftimie Murgu nr. 2, 300041 Timișoara, Romania. Tel: +40 256204400 Anca Dinu • Piata Eftimie Murgu nr. 2, 300041 Timișoara, Romania. Tel: +40 256204400 Ovidiu Horea Bedreag • Piata Eftimie Murgu nr. 2 300041 Timișoara, Romania. Tel: +40 256204400 Sonia Elena Popovici • Piața Eftimie Murgu nr. 2 300041 Timișoara, Romania. Tel: +40 256204400 Alexandra Enache • Piața Eftimie Murgu nr. 2, 300041 Timișoara, Romania. Tel: +40 256204400

\begin{abstract}
The current practice in the field of forensic medicine imposes the use of modern investigation techniques. The complexity of laboratory investigation methods needed for a final result of the investigation in forensic medicine needed new biomarkers of higher specificity and selectivity. Such biomarkers are the microRNAs (miRNAs), short, non-coding RNAs composed of 19-24 nucleotides. Their characteristics, such as high stability, selectivity, and specificity for biological fluids, differ from tissue to tissue and for certain pathologies, turning them into the ideal candidate for laboratory techniques used in forensic medicine. In this paper, we wish to highlight the biochemical properties and the usefulness of miRNAs in forensic medicine.
\end{abstract}

Keywords: miRNA expression, forensic biomarkers, noninvasive detection

\section{INTRODUCTION}

In recent years, notable progress has been made regarding identification, analysis, and investigation methods in forensic sciences. ${ }^{1,2}$ In order to obtain an efficient and accurate result, precise identification methods are needed for certain factors that are meant to answer a series of questions. This is the reason the analysis of microRNA (miRNA) expression represents a good tool for the future, to be introduced in the forensic laboratory. ${ }^{1,3,4}$

miRNAs are short, non-coding RNAs that contain 19-24 nucleotides. From a historical point of view, they were isolated and analyzed for the first time in 1990 in Caenorhabditis elegans. ${ }^{5}$ The utility of miRNAs in medical practice is based on certain characteristics they encompass, such as high resistance to external factors, specificity for a certain fluid or tissue, specificity for a certain disease, selectivity, and, last but not least, low processing costs. ${ }^{6}$ At the same time, miRNAs are involved in different pathological changes for which they present high selectivity and specificity such as cancer, functional imbalance, infections, or traumatisms. 
These properties make miRNAs the ideal candidate for usage in the routine practice of the forensic laboratory. ${ }^{1,7}$

For a more efficient analysis of miRNAs, different analysis techniques were developed. The most well-known identification, isolation, and characterization methods for miRNAs are quantitative real-time PCR (qPCR), miRNA arrays, RNA sequencing, or multiplex miRNA profiling. ${ }^{8-12}$

In the following paper, we wish to present the biological and biochemical characteristics of miRNAs and their utility in the forensic laboratory, in accordance with their specificity and selectivity for certain biological fluids, tissues, or diseases.

\section{BIOGENESIS OF MIRNAS}

The first stage in the biogenesis of miRNAs is represented by the transcription of RNA sequences by RNA polymerase II. ${ }^{13}$ This process leads to the formation of the first miRNAs, the so-called pri-miRNAs. The factors responsible for the transcriptional modulation of these precursors are represented by members of the TP53 family such as TFD, p53, p63, and p73. ${ }^{14,15}$ pri-miRNAs are RNA sequences composed of approximately 70 nucleotides. They are subject to the action of two proteins with nuclear activity, i.e. nuclear RNA-binding protein DiGeorge Syndrome Critical Region 8 (DGCR8) and ribonuclease III-type protein Drosha. The molecular reactions modulated by these two proteins lead to the formation of pre-miRNAs, the precursors of miRNAs, followed by their export from the nucleus into the cytoplasm through a nuclear transport protein called Exportin 5. ${ }^{16,17}$ In the cytoplasm, the pre-miRNAs are transformed into the miRNA/miRNA* duplex through the action of a Dicer protein. A fragment of this duplex forms a complex with the RNA-induced silencing complex (RISC), this being the last stage in the biogenesis of mature miRNAs, composed of 19-24 nucleotides. ${ }^{18,19}$

Some of the miRNAs are exported from the cell in different ways. There are two release mechanisms discussed in the literature, active and passive. In the case of active release mechanisms, miRNAs are attached to cellular secretions such as lipoproteins, micro-vesicles, exosomes, and ectosomes, while in the case of passive release mechanisms they end up in the extracellular environment in the form of apoptotic bodies ${ }^{20-22}$ (Figure 1).

\section{SPECIFICITY OF MIRNAS FOR BIOLOGICAL FLUIDS AND TISSUES}

Because the cell is capable to release miRNAs in the extracellular space, their impact on different areas and lines of work such as medicine, criminalistics, and diagnostic laboratories has been discussed in a series of specialty papers in the literature. Moreover, the utility of miRNAs in analysis and diagnosis methods took wings in recent years due to their specificity for certain types of tissues and fluids. ${ }^{6}$ Recent studies report a series of specific miRNAs, either for certain tissues, or for certain biological fluids. Wang et $a l$. isolated and identified miRNA expressions in different biological fluids such as venous blood, vaginal discharge, menstrual blood, sperm, saliva, and buccal swabs, using the TaqMan RT-qPCR technique. Their studies showed a high specificity of miRNA-16 for venous blood, in comparison to other fluids, turning it into an important tool for forensic medicine and criminalistics. ${ }^{4}$

Weber et al. conducted a similar study, analyzing 12 biological fluids - amniotic fluid, breast milk, bronchial lavage, cerebrospinal fluid, colostrum, peritoneal fluid, saliva, seminal fluid, tears, and urine - in order to obtain a profile of constitutive miRNA expression..$^{23}$ They reported hundreds of identified miRNAs for each fluid type, and in order to determine miRNA specificity for each fluid type, they performed further validation tests for these certain species. By using the validation methods for miRNA, they reported a series of miRNAs with high specificity for a certain type of biological fluid. They identified miRNA-224, miRNA-483-3p, miRNA-518f*, miRNA-508-3p, miRNA551b, miRNA-182, miRNA-135a*, miRNA-139-3p, miRNA-801, miRNA-369-3p, miRNA-801, miRNA-369-3p, miRNA-519d, miRNA-229-5p, miRNA-373, miRNA330-5p; for breast milk miRNA-193b, miRNA-10a, miRNA-28-5p, miRNA-924, miRNA-150*, miRNA-518c*, miRNA-217; for saliva miRNA-182*, miRNA-450b-5p, miRNA-622, miRNA-141, miRNA-26a, miRNA-145*, miRNA-135b*, miRNA-381, miRNA-96*, miRNA-1228, miRNA-431*; for peritoneal fluid miRNA-129*, miRNA-583, miRNA-223, miRNA-627, miRNA-29b-1*; for amniotic fluid miRNA-636, miRNA-92a- ${ }^{*}$, miRNA376b, miRNA-26b, miRNA-556-5p, miRNA-593*; for seminal fluid miRNA-508-5p, miRNA-644, miRNA-17, miRNA-380*, miRNA-29b-2*, miRNA-340; and for tears miRNA-637. They also identified miRNAs species in colostrum such as miRNA-18a*, miRNA-513-5p, miRNA-10b*, miRNA-192*, miRNA-193b*, miRNA-130a*, while in the cerebrospinal fluid miRNA-577. ${ }^{23}$

Petersen et al. also studied miRNA expression in blood samples, saliva, and sperm. After several determinations, they identified a series of miRNAs such as miRNA-4301, miRNA-451, miRNA-486-5p, miRNA-223, miRNA-29c, miRNA-4286, miRNA-16, miRNA-205, miRNA-1246, and miRNA-1274a. ${ }^{3}$ Hanson et al. conducted a similar study in 


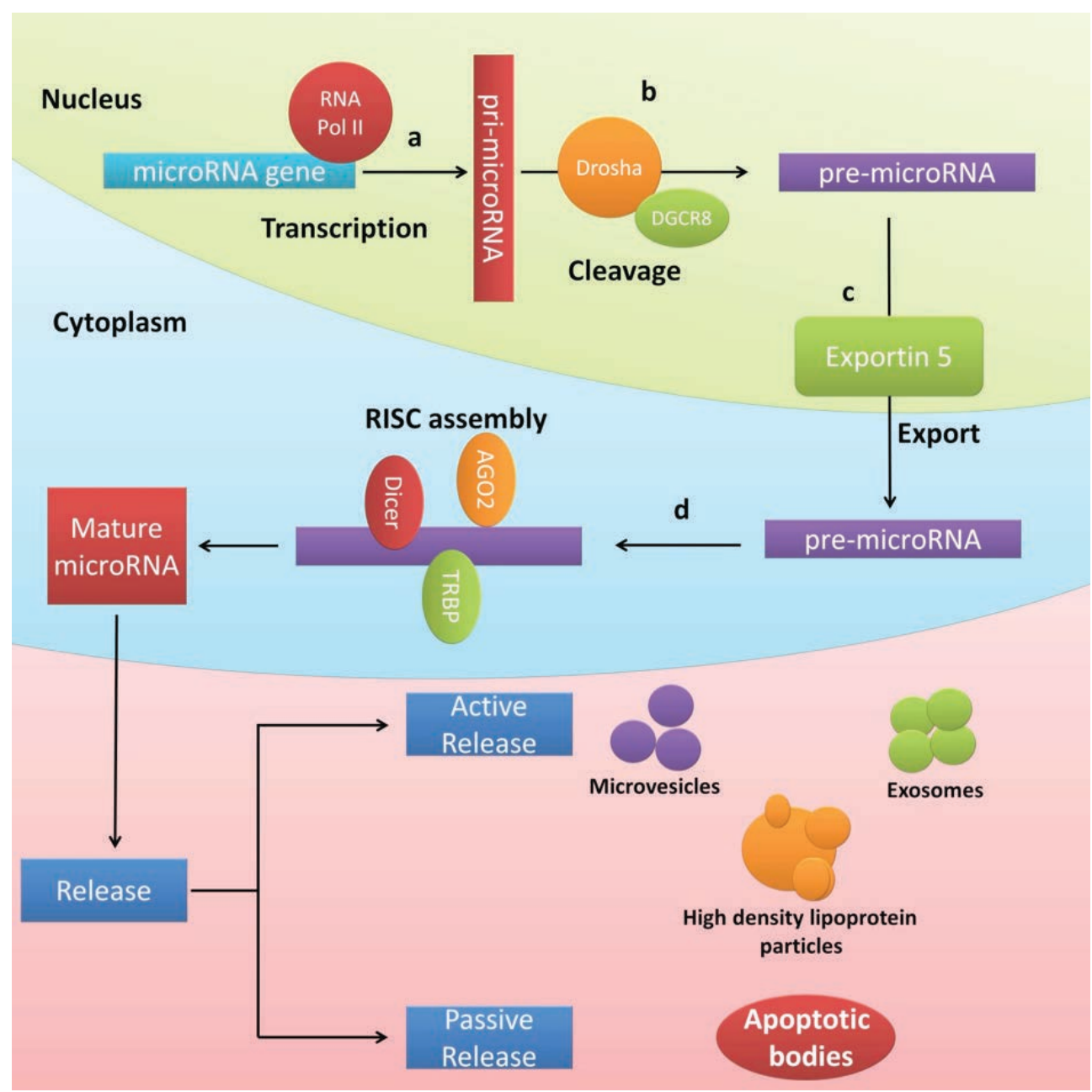

FIGURE 1. Schematic representation of miRNAs biogenesis. Reproduced after Dumache et al.22

which they analyzed the expression of miRNAs in different biological fluids, underlining the specificity for each type of fluid, which is of utmost importance for their usage in forensic medicine. They identified a series of species with high specificity for certain fluids like blood with miRNA-16 and miRNA-451, semen with miRNA-135b and miRNA-10b, saliva with miRNA-205 and miRNA-658, vaginal secretion miRNA-124a and miRNA-372, and menstrual blood with miRNA-451 and miRNA-412. ${ }^{24}$

With regard to miRNA specificity for certain tissues, a series of miRNA expressions were reported in the literature, which can be used in forensic medicine and criminal investigations. Guo et al. studied miRNA expression presenting specificity for a range of tissues, identifying miRNAs with high specificity for certain organs and systems. ${ }^{25-29}$ For the heart they identified miRNA-1, miRNA-126, miRNA-208, miRNA-302a, miRNA-302b, miRNA-302c, miRNA-302d, miRNA-367, miRNA-133a, and miRNA-133b; for the kidney they reported expression of
miRNA-200a, miRNA-196a, miRNA-196b, miRNA-10a, miRNA-10b, miRNA-146a, miRNA-30c, and miRNA204; for the liver miRNA-122, miRNA-483, miRNA-92a, and miRNA-192. In the neural tissue they identified a variety of miRNAs, as follows: miRNA-199a, miRNA199b, miRNA-214, miRNA-153, miRNA-137, miRNA-7, miRNA-143, miRNA-99b, miRNA-125a, miRNA-125b, miRNA-31, miRNA-124, miRNA-129, miRNA-138, miRNA-218, miRNA-708, miRNA-8, miRNA-128a, miRNA128b, miRNA-186, miRNA-95, miRNA-149, miRNA-323, miRNA-330, miRNA-33a, miRNA-346, miRNA-93, and miRNA-212. Also, there were certain miRNA expressions identified for the spleen - miRNA-223 and miRNA-146a; for the thymus - miRNA-96, miRNA-182, and miRNA-205; for bones - miRNA-482, miRNA-377, and miRNA-92a; or for the pancreas - miRNA-216a, miRNA216b, and miRNA-217. ${ }^{30}$ Sood et al. have studied miRNA expression in body tissues, reporting the existence of correlations between a certain tissue type and miRNA expres- 
sion in certain species. ${ }^{30}$ For the liver they have identified the expression of miRNA-122a, for the heart miRNA-1 and miRNA-133a, for the brain miRNA-9, for the pancreas miRNA-216, for the lungs miRNA-223, for testis miRNA-204, for bone marrow miRNA-223, and for skeletal muscle miRNA- 1 and miRNA- $133^{\circ} .30-35$

\section{MIRNAS IN TOXICOLOGY}

A high number of investigations in forensic medicine and criminalistics have as main aim the identification of potential toxic substances. While most toxic substances act at cellular level, they also have an influence on the molecular mechanisms involved in the biogenesis of miRNAs. This led to the identification of a series of miRNAs with high specificity for certain toxic substances.

Yang et al. have studied the modifications induced by acetaminophen in children. Their studies reported a significant increase in plasma miRNAs such as miRNA-122, miRNA-375, miRNA-423-5p, miRNA-30d-50, miRNA-125b-5p, miRNA-4732-5p, miRNA-204-5p, miRNA-574-3p, as well as urine miRNAs such as miRNA-375, miRNA-940, miRNA-9-3p, and miRNA-302a. ${ }^{36}$ Wang et al. have studied the toxic effects induced by four volatile agents, formaldehyde, benzene, toluene, and xylene, on miRNA expression. Their studies underline significant changes in miRNA-1187, miRNA-125a-3p, miRNA-125b-5p, miRNA-466c-5p, miRNA-5105, and miRNA-3472 expressions. ${ }^{37}$ A similar study was conducted by Bollati et al. regarding the influence of cadmium on miRNA-146a expression. 38,39

Bisphenol $\mathrm{A}$ is a compound that plays an essential role in the production of plastic masses used in the fast-food industry. Awissar-Whiting et al. have reported changes in the expression of miRNA-21, miRNA-335, and miRNA-153 after interactions with this compound. ${ }^{40}$

Another compound with toxic actions is 2,3,7,8-tetrachlorodibenzo-p-dioxin, found in tobacco leafs. Singh et $a l$. have studied the effects this compound has on miRNA expression, reporting important changes in the expression of miRNA-122, miRNA-181a, miRNA-23a, miRNA-18b, miRNA-31, and miRNA-182. ${ }^{41}$

\section{THE USE OF mIRNAS IN AUTOPSY}

The use of miRNA expression can bring a variety of improvements to the analysis methods used in the forensic autopsy. Whereas, in the literature, miRNA expression it associated with a series of pathologies and pathophysiological dysfunctions, it can be a good indicator in clarifying the cause of death.

\section{USING MIRNAS AS A BIOMARKER FOR CANCER}

Identifying miRNA expression has lately gained momentum especially due to research conducted in neoplastic diseases. Because cancer patients need fast, cheap, and precise diagnostic methods, miRNA expression and their correlations with a series of clinical aspects have been studied extensively in these patients. Recent studies have shown that there are significant correlations between specific modifications of miRNA expressions and certain types of cancer.

Gastric cancer is one of the most common types of cancer, being responsible for a high number of deaths around the world. Liu et al. have identified an increase in miRNA-1 expression in case of gastric cancer patients. ${ }^{42}$ Zhang et al. have also studied miRNA expression in gastric cancer, showing a significant decrease in miRNA-421 expression in this disease. ${ }^{43}$ Song et al. have reported an increase in miRNA-221 expression in gastric cancer patients. ${ }^{44}$ Another frequent type of cancer is colorectal cancer. Huang et al. have reported an increase in miRNA-29a expression in case of patients suffering from this disease. ${ }^{45}$

Lung cancer kills a large number of subjects every year. It is triggered either by a genetic predisposition, or exogenous factors. ${ }^{46}$ Wang et al. have studied miRNA expression in pulmonary cancer patients and showed a modification of miRNA-125a-5-, miRNA-25, and miRNA-126 in sick individuals, in comparison to healthy ones. Moreover, their study underlined a higher than $80 \%$ specificity and sensitivity for these miRNAs in pulmonary cancer. ${ }^{47}$

Rehbein et al. have also conducted research regarding miRNA expression in bronchial lavage, identifying a series of changes in miRNA-19b-1, miRNA-1285, miRNA-1289, miRNA-1303, miRNA-217, miRNA-29a-5p, miRNA548-3p, and miRNA-650 expressions. ${ }^{48}$ Powrozek et al. also identified significant changes in miRNA-944 and miRNA-3662 expressions in lung cancer. ${ }^{49}$

If we were to have a global view on the molecular changes induced by oncological diseases, we can say that for each type of cancer there is - or should be - a specific type of miRNA or a certain miRNA with higher specificity. Clearly, there has been extensive research on specific miRNAs in different types of cancer, more than what we have mentioned so far. Thus, Zhou et al. identified changes in miRNA-21, miRNA-26a, miRNA-27a, miRNA-122, miRNA-192, miRNA-223, and miRNA-801 in case of hepatocellular cancer. ${ }^{50}$ Vincent et al. report changes for miRNA-16 and miRNA-25 in case of multiple myeloma. ${ }^{51}$ Mitchell et al. have studied miRNA expression in case of prostate cancer patients and have shown significant chang- 
es in miRNA-141 expressions..$^{52}$ In a similar study, Redova et al. acknowledge miRNA-141 modifications in case of prostate cancer. ${ }^{53}$

\section{USE OF MIRNAS AS A BIOMARKER FOR CERTAIN CLINICAL DISORDERS}

There are a series of lethal clinical disorders. Using miRNAs for their diagnosis can be useful in establishing the cause of death during the autopsy. Moreover, highlighting specific miRNAs for each type of affection can facilitate the work of the forensic pathologist, being of utmost help in forensic medicine.

Cardiovascular diseases are a leading cause of death worldwide, ${ }^{54}$ and coronary artery disease and its complication, acute myocardial infarction, are the most frequently discussed diseases in the literature. Recent studies report a series of modifications at the miRNA level in case of coronary artery disease. Fictlscherer et al. have studied the modifications of miRNA expressions in case of cardiovascular diseases and especially coronary diseases. ${ }^{55}$ Their study shows a significant decrease in miRNA-126, miRNA-17, miRNA-92a, miRNA-145, and miRNA-155 and an increase in miRNA-133 and miRNA-208a expressions. ${ }^{55}$ In a similar study, Weber et al. identified an important decrease of miRNA-19a, miRNA-484, miRNA-155, miRNA-222, miRNA-29a, miRNA-378, miRNA-342, miRNA-181a, miRNA-145, miRNA-150, and miRNA-20e-5p in case of patients suffering of coronary artery disease..$^{56}$

Acute myocardial infarction is also one of the leading causes of death. Cheng et al. identified an increase in miRNA-1 expression in these patients, ${ }^{57}$ their findings being confirmed by Ai et al. ${ }^{8}$ Similar studies have been conducted by Olivieri et al., reporting an increase in miRNA-1, miRNA-133a, miRNA-423-5p, miRNA-499-5p, and miRNA-21 expressions in myocardial infarction. ${ }^{59}$

miRNAs can also be used in the diagnosis of a variety of other diseases such as Alzheimer's. Cogswell et al. have studied changes in miRNA expressions in this disease, reporting a decrease in miRNA-127, miRNA-181c, miRNA-9, and miRNA-29a/b expressions. ${ }^{60}$ Another common affection is diabetes mellitus. Karoline et al. have identified an increase in miRNA-144 expression in these patients.

\section{USING MIRNAS AS A BIOMARKER FOR MULTIPLE TRAUMA}

Multiple trauma can occur either after car or domestic accidents, or after physical aggression. In order to facilitate tissue, organ, or system diagnosis, several studies regard- ing miRNA expression in case of trauma have been conducted. ${ }^{61}$

Traumatic brain injury is one of the most common traumatisms, being responsible for severe disability. Wang et al. have analyzed the expression of miRNAs in case of cerebral injuries, highlighting an increase in miRNA-155 and miRNA-223 expression. ${ }^{62}$ In a similar study conducted by Sun $e$ al., a significant increase in miRNA-142-3p and miRNA-221 expression in case of severe brain injuries has been reported.63

Spinal cord injury is of similar severity. ${ }^{64}$ Liu et al. have shown a decrease in miRNA-137, miRNA-181a, miRNA219-2-3p, and miRNA-7a expression and an increase in microRNA-21 expression in case of laboratory models with induced spinal cord injuries. ${ }^{65}$

In case of a multiple trauma, the thoracic injuries are most common, leading indirectly to respiratory failure. This condition appears due to direct or secondary injuries, including severe inflammation, infections, or acute respiratory distress syndrome (ARDS). Huang et al. have analyzed miRNA expression in case of laboratory animals with ARDS and have shown an increase in miRNA-99a, miRNA-344, miRNA-127, miRNA-346, miRNA-128b, miRNA135b, miRNA-30a, and miRNA-30b expression. They have also identified a decreased expression of miRNA-26a, miRNA-126, and miRNA-24. 66

\section{USING MIRNAS AS A BIOMARKER IN SEVERE INFECTIONS}

A large number of subjects die every year because of severe infections. ${ }^{67}$ In current medical practice, there are a number of diagnostic techniques to identify the pathogen. Nevertheless, there are situations in which patients are in a critical stage because of pathophysiological and biochemical dysfunctions, severe inflammation, immunodepression, and multiple injuries, and these patients eventually develop sepsis. Because of infections caused by multiple germs, but also because of the constant decrease in the body's immune capacity, septic shock will appear, leading to multiple organ dysfunction syndrome (MODS). Unfortunately, a high percentage of patients die every year because of severe infections and MODS.

Lately, there have been a series of modifications induced by pathogens on miRNA expression, representing a real challenge for forensic medicine. Wang et al. have shown a decrease in miRNA-223 and miRNA-146 expression in case of septic patients. ${ }^{68}$ Vasilescu et al. have made a statistically significant correlation between miRNA-150 and an increase in the concentration of pro-inflammatory 
cytokines such as interleukin 18 (IL-18). ${ }^{69}$ Schmidt et al. analyzed the influence of Escherichia coli on miRNA expression, showing changes in miRNA-146b, miRNA-150, miRNA-143, miRNA-342, and miRNA-143 expression. ${ }^{70}$

\section{CONCLUSIONS}

In forensic sciences, the identification of the type and origin of body fluids (saliva, whole blood, menstrual blood, semen, vaginal secretions, and urine) found at the crime scene represents an important tool for the identification of the biological source. At present, conventional serological and biochemical methods used for the identification of body fluids have certain limitations such as low sensitivity and specificity, time and sample consumption, and laboratory work. In the last 20 years, DNA molecular analysis has become an important tool in forensic investigations. Currently, it is possible to genotype all types of biological traces or micro-traces containing nucleated cells if they are not entirely destroyed chemically or by bacteria. DNA profiling is based on short tandem repeats (STR) and aids in the identification of biological samples, but due to the recent advances in molecular genetics over the past 10 years, new methods have been developed and introduced, and other biomarkers, such as miRNAs, have been proposed to be used in forensic identifications.

Introducing miRNAs in the current forensic laboratory practice represents a golden goal regarding investigation, monitoring, and diagnostic methods. Their high specificity, as well as their compatibility with DNA analysis, makes them 'ideal' biomarkers for forensic medicine use. At the same time, research needs to be continued in order to assess the performance of microRNAs as biomarkers in determinations from degraded samples or degraded biological fluids.

In conclusion, we can state that miRNAs can be used as biomarkers in the diagnosis of a variety of pathophysiological dysfunctions or as indicators in forensic medicine procedures. Nevertheless, more studies are needed regarding the specificity and selectivity of miRNAs for biological fluids, tissues, or a series of pathophysiological disorders.

\section{CONFLICT OF INTEREST}

Nothing to declare.

\section{REFERENCES}

1. Hanson EK, Lubenow H, Ballantyne J. Identification of forensically relevant body fluids using a panel of differentially expressed microRNAs. Anal Biochem. 2009;387:303-314.
2. Verduijn R. Recent Developments in Forensic Biology. BSc Biol. 2010

3. Petersen $\mathrm{CH}$, Hjort BB, Tvedebrink T, Kielpinski LJ, Vinther J, Morling N. Body fluid identification of blood, saliva and semen using second generation sequencing of micro-RNA. Forensic Sci Int Genet Supp/ Ser. 2013:4:e204-e205.

4. Wang Z, Zhang J, Luo H, Ye Y, Yan J, Hou Y. Screening and confirmation of microRNA markers for forensic body fluid identification. Forensic Sci Int Genet. 2013;7:116-123.

5. Meng L, Chen L, Li Z, Wu Z-X, Shan G. Roles of microRNAs in the Caenorhabditis elegans nervous system. J Genet Genomics. 2013;40:445452.

6. Zubakov D, Boersma AWM, Choi Y, Van Kuijk PF, Wiemer EC, Kayser M. MicroRNA markers for forensic body fluid identification obtained from microarray screening and quantitative RT-PCR confirmation. Int J Legal Med. 2010;124:217-226.

7. Wang Z, Luo H, Pan X, Liao M, Hou Y. A model for data analysis of microRNA expression in forensic body fluid identification. Forensic Sci Int Genet. 2012;6:419-423.

8. Meng S, Cao JT, Zhang B, Zhou Q, Shen CX, Wang CQ. Downregulation of microRNA-126 in endothelial progenitor cells from diabetes patients, impairs their functional properties, via target gene Spred-1. J Mol Cell Cardiol. 2012;53:64-72

9. Guo HQ, Huang GL, Guo CC, Pu XX, Lin TY. Diagnostic and prognostic value of circulating miR-221 for extranodal natural killer/T-cell lymphoma. Dis Markers. 2010;29:251-258.

10. Deng H, Guo Y, Song H, et al. MicroRNA-195 and microRNA-378 mediate tumor growth suppression by epigenetical regulation in gastric cancer. Gene. 2013;518:351-359.

11. Uhlich RM, Konie JA, Davis JW, et al. Novel microRNA correlations in the severely injured. Surgery. 2014;156:834-840.

12. Liu T, Cheng W, Gao Y, Wang H, Liu Z. Microarray analysis of microRNA expression patterns in the semen of infertile men with semen abnormalities. Mol Med Rep. 2012;6:535-542.

13. Chen W, Harbeck MC, Zhang W, Jacobson JR. MicroRNA regulation of integrins. Trans/ Res. 2013;162:133-143.

14. Abdelmohsen K, Srikantan S, Kang MJ, Gorospe M. Regulation of senescence by microRNA biogenesis factors. Ageing Res Rev. 2012;11:491500.

15. Westholm JO, Lai EC. Mirtrons: microRNA biogenesis via splicing. Biochimie. 2011:93:1897-1904.

16. Von Brandenstein M, Richter C, Fries JWU. MicroRNAs: Small but amazing, and their association with endothelin. Life Sci. 2012;91:475-489.

17. Yi R, Qin Y, Macara IG, Cullen BR. Exportin-5 mediates the nuclear export of pre-microRNAs and short hairpin RNAs Exportin-5 mediates the nuclear export of pre-microRNAs and short hairpin RNAs. Genes Dev. 2003:17:3011-3016.

18. Cheng G. Circulating miRNAs: roles in cancer diagnosis, prognosis and therapy. Adv Drug Deliv Rev. 2015;81:75-93.

19. Andersen HH, Duroux M, Gazerani P. MicroRNAs as modulators and biomarkers of inflammatory and neuropathic pain conditions. Neurobiol Dis. 2014;71:159-168.

20. Weilner S, Schraml E, Redl H, Grillari-Voglauer R, Grillari J. Secretion of microvesicular miRNAs in cellular and organismal aging. Exp Gerontol. 2013;48:626-633

21. Rana S, Malinowska K, Zöller M. Exosomal tumor microRNA modulates premetastatic organ cells. Neop/asia. 2013;15:281-295.

22. Dumache R, Rogobete AF, Bedreag $\mathrm{OH}$, et al. Use of miRNAs as biomarkers in sepsis. Anal Cell Pathol (Amst). 2015;2015:186716.

23. Weber J, Baxter DH, Zhang S, et al. The MicroRNA Spectrum in 12 Body Fluids. Clin Chem. 2010;56:1733-1741.

24. Hanson $\mathrm{E}$, Lubenow $\mathrm{H}$, Ballantyne J. Identification of forensically relevant body fluids using a panel of differentially expressed microRNAs. Forensic Sci Int Genet Supp/ Ser. 2009;2:503-504.

25. Williams G, Uchimoto ML, Coult N, World D, Beasley E, Avenell P. Characterisation of body fluid specific microRNA markers by capillary electrophoresis. Forensic Sci Int Genet Supp/ Ser. 2013;4:e274-e275.

26. Kuner R, Brase JC, Sültmann H, Wuttig D: microRNA biomarkers in body fluids of prostate cancer patients. Methods. 2013;59:132-137.

27. Gilad S, Meiri E, Yogev Y, et al. Serum microRNAs are promising novel biomarkers. PLoS One. 2008;3:1-7.

28. Roth C, Rack B, Müller V, Janni W, Pantel K, Schwarzenbach H. Circulating microRNAs as blood-based markers for patients with primary and metastatic breast cancer. Breast Cancer Res. 2010;12:R90. 
29. Guo Z, Maki M, Ding R, Yang Y, Zhang B, Xiong L. Genome-wide survey of tissue-specific microRNA and transcription factor regulatory networks in 12 tissues. Sci Rep. 2014;4.

30. Sood P, Krek A, Zavolan M, Macino G, Rajewsky N. Cell-type-specific signatures of microRNAs on target mRNA expression. Proc Natl Acad Sci USA. 2006:103:2746-2751.

31. Bloomston M, WL F, Petrocca F, et al. MicroRNA expression patterns to differentiate pancreatic adenocarcinoma from normal pancreas and chronic pancreatitis. JAMA. 2007;297:1901-1908.

32. Tsai HL, Yang IP, Huang CW, et al. Clinical significance of microRNA-148a in patients with early relapse of stage II stage and III colorectal cancer after curative resection. Trans/ Res. 2013;162:258-268.

33. Harraz MM, Dawson TM, Dawson VL. MicroRNAs in Parkinson's disease. J Chem Neuroanat. 2011;42:127-130.

34. Papurica M, Rogobete AF, Sandesc D, et al. Advances in Biomarkers in Critical III Polytrauma Patients. Clin Lab. 2016;62:977-986.

35. Jones K, Nourse JP, Keane C, Bhatnagar A, Gandhi MK. Plasma microRNA are disease response biomarkers in classical hodgkin Iymphoma. Clinical Cancer Research. 2014;20:253-264.

36. Yang X, Salminen WF, Shi Q, et al. Potential of extracellular microRNAs as biomarkers of acetaminophen toxicity in children. Toxicol Appl Pharmacol. 2015;284:180-187.

37. Wang F, Li C, Liu W, Jin Y. Effect of exposure to volatile organic compounds (VOCs) on airway inflammatory response in mice. J Toxicol Sci. 2012;37:739-748.

38. Cao Y, Yu SL, Wang Y, Guo GY, Ding Q, An RH. MicroRNA-dependent regulation of PTEN after arsenic trioxide treatment in bladder cancer cell line T24. Tumor Biol. 2011;32:179-188.

39. Bollati $\vee$, Marinelli $B$, Apostoli $P$, et al. Exposure to metal-rich particulate matter modifies the expression of candidate MicroRNAs in peripheral blood leukocytes. Environ Health Perspect. 2010;118:763-768.

40. Avissar-Whiting M, Veiga KR, et al. Bisphenol A exposure leads to specific microRNA alterations in placental cells. Reprod Toxicol. 2010;29:401-406.

41. Singh NP, Singh UP, Guan H, Nagarkatti P, Nagarkatti M. Prenatal Exposure to TCDD Triggers Significant Modulation of microRNA Expression Profile in the Thymus That Affects Consequent Gene Expression. PLoS One. 2012; 7:e45054

42. Liu R, Zhang C, Hu Z, et al. A five-microRNA signature identified from genome-wide serum microRNA expression profiling serves as a fingerprint for gastric cancer diagnosis. Eur J Cancer. 2011;47:784-791.

43. Zhang X, Cui L, Ye G, et al. Gastric juice microRNA-421 is a new biomarker for screening gastric cancer. Tumor Biol. 2012;33:2349-2355.

44. Song MY, Pan KF, Su HJ, et al. Identification of serum microRNAs as nove non-invasive biomarkers for early detection of gastric cancer. PLoS One. 2012;7:1-9.

45. Huang Z, Huang D, Ni S, Peng Z, Sheng W, Du X. Plasma microRNAs are promising novel biomarkers for early detection of colorectal cancer. Int $J$ Cancer. 2010;127:118-126.

46. Zhu C, Tsao M. Prognostic markers in lung cancer: is it ready for prime time? Trans/ Lung Cancer Res. 2014;3:149-158.

47. Wang P, Yang D, Zhang $H$, et al. Early Detection of Lung Cancer in Serum by a Panel of MicroRNA Biomarkers. Clin Lung Cancer. 2014;16:313-319.e1

48. Rehbein G, Schmidt B, Fleischhacker M. Extracellular microRNAs in bronchoalveolar lavage samples from patients with lung diseases as predictors for lung cancer. Clin Chim Acta. 2015;450:78-82.

49. Powrózek T, Krawczyk P, Kowalski DM, Winiarczyk K, Olszyna-Serementa M, Milanowski J. Plasma circulating microRNA-944 and microRNA-3662 as potential histologic type-specific early lung cancer biomarkers. Transl Res. 2015;166:315-323.

50. Zhou J, Yu L, Gao X, et al. Plasma MicroRNA Panel to Diagnose Hepatitis B Virus-Related Hepatocellular Carcinoma. J Clin Oncol. 2011;29:4781-4788.

51. Vincent L, Vang D, Nguyen J, et al. Mast cell activation contributes to sickle cell pathobiology and pain in mice. Blood. 2013;122:1853-1862.

52. Mitchell PS, Parkin RK, Kroh EM, et al. Circulating microRNAs as stable blood-based markers for cancer detection. Proc Natl Acad Sci USA. 2008:105:10513-10518.

53. Redova M, Sana J, Slaby O. Circulating miRNAs as new blood-based biomarkers for solid cancers. Futur Oncol. 2013;9:387-402.

54. Lavie L, Lavie P. Molecular mechanisms of cardiovascular disease in OSAHS: The oxidative stress link. Eur Respir J. 2009;33:1467-1484.

55. Fichtlscherer S, De Rosa S, Fox H, et al. Circulating microRNAs in patients with coronary artery disease. Circ Res. 2010;107:677-684.

56. Weber M, Baker MB, Patel RS, Quyyumi AA, Bao G, Searles CD. MicroRNA Expression Profile in CAD Patients and the Impact of ACEl/ARB. Card Res Pract. 2011;2011:532915

57. Cheng Y, Tan N, Yang J, et al. A translational study of circulating cell-free microRNA-1 in acute myocardial infarction. Clin Sci (Lond). 2010;119:87-95.

58. Ai J, Zhang R, Li Y, et al. Circulating microRNA-1 as a potential novel biomarker for acute myocardial infarction. Biochem Biophys Res Commun. 2010;391:73-77.

59. Olivieri F, Antonicelli R, Lorenzi M, et al. Diagnostic potential of circulating miR-499-5p in elderly patients with acute non ST-elevation myocardial infarction. Int J Cardiol. 2013;167:531-536.

60. Cogswell JP, Ward J, Taylor IA, et al. Identification of miRNA changes in Alzheimer's disease brain and CSF yields putative biomarkers and insights into disease pathways. J Alzheimers Dis. 2008;14:27-41.

61. Karolina DS, Armugam A, Tavintharan S, et al. MicroRNA 144 impairs insulin signaling by inhibiting the expression of insulin receptor substrate 1 in type 2 diabetes mellitus. PLoS One. 2011;6.

62. Bedreag OH, Sandesc D, Chiriac SD, et al. The Use of Circulating miRNAs as Biomarkers for Oxidative Stress in Critically III Polytrauma Patients. Clin Lab. 2016;62:263-274.

63. Sun T, Chen X, Liu Z, et al. Expression profiling of MicroRNAs in hippocampus of rats following traumatic brain injury. J Huazhong Univ Sci Technol Medical Sci. 2014:34:548-553.

64. Butcher N, Balogh ZJ. The definition of polytrauma: the need for international consensus. Injury. 2009;40:S12-S22.

65. Liu NK, Wang XF, Lu B, Xu XM. Altered microRNA expression following traumatic spinal cord injury. Exp Neurol. 2009;219:424-429.

66. Huang C, Xiao X, Chintagari NR, Breshears M, Wang Y, Liu L. MicroRNA and mRNA expression profiling in rat acute respiratory distress syndrome. BMC Med Genomics. 2014;7:1-15.

67. Ndegwa LK, Katz MA, McCormick K, et al. Surveillance for respiratory health care-associated infections among inpatients in 3 Kenyan hospitals. Am J Infect Control. 2014;42:985-990.

68. Wang J, Yu M, Yu G, et al. Serum miR-146a and miR-223 as potential new biomarkers for sepsis. Biochem Biophys Res Commun. 2010;394:184-188.

69. Vasilescu C, Rossi S, Shimizu M, et al. MicroRNA fingerprints identify miR150 as a plasma prognostic marker in patients with sepsis. PLoS One. 2009; 4:e7404.

70. Schmidt WM, Spiel AO, Jilma B, Wolzt M, Müller M. In vivo profile of the human leukocyte microRNA response to endotoxemia. Biochem Biophys Res Commun. 2009;380:437-441. 